\title{
La décolonisation des femmes et de la nature
}

\author{
Exercices "sages " pour réfléchir à propos \\ des femmes et de l'écologie \\ Maria Inácia D’Avila Neto \\ Claudio Cavas \\ Gabriel Sena Jardim
}

\begin{abstract}
[Résumé] Les questions portant sur l'écologie politique féministe sont stimulantes pour l'examen des vieilles dichotomies qui insistent sur l'association fallacieuse de la femme avec la nature et de l'homme avec la culture. Le présent article accomplit un parcours visant à discuter des constructions identitaires des femmes et de l'appropriation de la nature, sillonné par des questions de genre, race et niveau socio-économique des femmes au Brésil, au travers de quelques perspectives des études culturelles et postcoloniales.
\end{abstract}

Mots-clés : femmes, nature, écologie politique, postcolonial, études culturelles, Brésil.

\section{Introduction}

Parmi les différents courants du féminisme et de l'écologie, y compris l'écoféminisme et le féminisme environnemental, on observe des avertissements à propos des erreurs et des pièges cherchant à associer la femme à la nature et l'homme à la culture. (Shiva, 2010; Mies et Shiva, 1999; Warren, 1987; Fraser, 2007; Butler, Spivak, 2007, Haraway, 1988; Bahri, 2006; Lugones, 2008; Miñoso, 2009; D’Ávila Neto, 2005, 2012). On trouve des critiques similaires dans la perspective des études postcoloniales et culturelles (Hall, 2006; Boaventura Santos, 2006), et dans la littérature latino-américaine traitant de la modernité et la colonialité1. La « décolonisation » du féminisme et des femmes y est recherchée, en essayant de démontrer " que les différentes formes de démocratie, des droits civiques et de l'émancipation des femmes ne peuvent provenir que de réponses créatives de la part des épistémologies subalternes locales ${ }^{2}$ (Grosfoguel 2008: 139). Ceci implique d'octroyer des possibilités d'expression aux « savoirs locaux »du point de vue de la construction identitaire des femmes et de limaginaire de la nature. Nous tenterons de tracer les voies de cette construction, en donnant des exemples de la société brésilienne, en utilisant des réflexions provenant des courants de l'écologie

${ }^{1}$ Néologisme pour colonialidade, mot utilisé en espagnol ; en portugais, colonialidade.

${ }^{2}$ Version en français des auteurs. 
politique féministe et de la littérature latino-américaine, tributaires des études culturelles et postcoloniales. Du point de vue de la discussion sur l'écologie politique féministe, l'approche écoféministe est vue par l'Américaine Donna Haraway, comme une importante contribution à une nouvelle " version du monde » : «les écoféministes ont peut-être été celles qui ont le plus travaillé à produire une version du monde comme sujet actif et non comme simple ressource cartographiée et confisquée par les projets bourgeois, marxistes ou mâles » (Haraway 2007 : 131). Karen Warren, résumant les points essentiels à propos de l'éthique écoféministe, déclare :

"Selon le féminisme écologique, il existe des liens importants entre les femmes, les personnes de couleur, les pauvres, et le non humain de la nature (...) le féminisme écologique trouve ses racines dans les différents féminismes (féminisme libéral, marxiste, socialiste, radical, Black feminism, féminisme du tiers-monde) (..) et ce qui distingue l'écoféminisme, c'est son insistance à considérer que la nature humaine et le naturisme, c'est-à-dire la domination non justifiée de la nature, sont des questions féministes. » (Warren 1993 : 191)

Par conséquent, l'écoféminisme ou, plus exactement, l'éthique écoféministe, rejette les aspects des écologistes profonds (deep ecology), en argumentant qu'ils n'atteignent pas le cœur du problème, à partir du moment où, bien que prenant comme postulat une vision anthropocentrique de la nature, ils le font de manière générique, mais en aucun cas, spécifique à la question masculine/féminine. Carolyn Merchant discute l'aspect épistémologique/philosophique de la question, dans son ouvrage La Mort de la Nature. Elle met en exergue le fait que, depuis le XVI ${ }^{\text {ème }}$ siècle, la destruction de l'idée d'un cosmos organisé, l'intégration des parties indépendantes entre le moi, la société et le cosmos, l'émergence d'un moi rationnel, dissociant le corps et l'âme, contribuent à l'approfondissement de la pensée dualiste au sein de laquelle, en recherchant de vieilles dichotomies, l'homme sera lié à la culture et la femme, à la nature et au corps (Ferry, L. 1992 ; D'Avila Neto, M.I., 1993, 1999).

\section{Invisibilités sociales comme vecteurs de naturalisation des femmes}

Notre objectif est de questionner la notion d'invisibilités sociales implicites qui renforcent la naturalisation des femmes et, par conséquent, des tâches qui leur sont « naturellement » attribuées, en prenant en compte la vie quotidienne des femmes brésiliennes. Cette naturalisation traite de la culture dominante et de ses propres présuppositions. Nous sommes conscients qu'à partir du moment où nous nous exprimons au sujet des femmes brésiliennes, ou même latino-américaines, nous faisons référence à de multiples univers ou à différentes manières d'être/connaître, sillonnés par la race/couleur de peau/classe/appartenance à des cultures locales.

La première invisibilité sociale observée se réfère au travail domestique, discussion persistante dans les mouvements féministes de différentes tendances. De fait, le discours de l'homogénéisation autour du travail des femmes semble avoir eu le plus de 
force, parce qu'il se fonde sur le «naturel » de la femme, c'est-à-dire dans ce qui serait sa nature intrinsèque, même si l'on admet que cette nature a été ingénieusement construite. Il devient alors nécessaire de comprendre que parler dans ce discours de la nature des femmes constitue également une supposition : celle qui veut que le corps des femmes soit destiné principalement à la reproduction de l'espèce. Ainsi, la femme est destinée au rôle de génitrice et de "personne qui prend soin », ce qui favorise la production d'un travail invisible, tel que les tâches domestiques, choses que « seule une femme sait faire » (D’Ávila Neto \& Nazareth 2005). Dans La société du Risque, Ulrich Beck attire l'attention sur le fait que la société industrielle a apporté la contre-modernité au sein de la propre modernité, ce qui se reflète dans le travail assigné aux femmes, invisible, mais plus qu'indispensable au maintien et au fonctionnement de la société industrielle. Un fait, tel que celui-ci, marque ainsi les limites entre masculin et féminin, au sein de la famille nucléaire, à partir du moment où l'exercice de l'activité professionnelle masculine s'appuie directement sur le travail domestique féminin. Alors que la production suit les lois du marché, au sein duquel les relations de travail sont de natures contractuelles, dans la sphère domestique, les relations sont appelées par Beck le « communautarisme collectif et familial du couple ». Selon lui, une prétention à l'égalité est impossible, à partir du moment où les structures socio-économiques disposent d'une base inégale comme condition sine qua non de son existence (D'Ávila Neto \& Nazareth 2005).

La connotation donnée aux activités domestiques des femmes, comme étant naturelles ou propres à la nature féminine, ne peut être seulement expliquée par sa description matérialisée. Quand les hommes cuisinent, que ce soit en tant que « loisir » ou que profession, ils peuvent devenir de grands chefs, sans qu'une étiquette d'activité féminine n'y soit collée. Lorsque les femmes assurent les mêmes tâches, en dehors du cadre d'une activité professionnelle, en ayant pour objectif la subsistance du foyer, ou même lorsqu'elles prennent soin des enfants, ou de personnes âgées, ces activités ont un caractère «naturel », propre à la femme. Il n'existe aucun prestige particulier associé à la réalisation de ces tâches, mais, bien au contraire, le fait de constater leur absence ou leur mauvaise réalisation, devient un "sujet moral » de désapprobation sociale. La femme est naturellement considérée, dans ce cas, comme une " personne prenant soin », et c'est cette caractéristique qui confère le caractère " naturel » de ses tâches et non pas ses descriptions matérielles. C'est un point important, lorsqu'il s'agit de comprendre les plaintes des « féministes du sud » contre l'homogénéisation du féminisme « occidental-blanc ». Il faut réfléchir sur ce que signifie, dans chaque culture, l'adoption, ou non, d'une nature maternelle de « personne prenant soin ». Dans le cadre d'une recherche réalisée en 2002, les femmes d'une association de brodeuses d'une région agricole du Brésil, nous ont montré, en tant que gardiennes d'enfants et de femmes au foyer, des visions idéalisées par des normes élaborées selon des critères moraux, souvent distantes de la pratique quotidienne des lourds travaux agricoles qu'elles réalisaient dans les champs, depuis leur plus jeune âge. Afin de recueillir les ressentis de ces 
femmes sur leurs propres expériences de terrain, un enregistrement vidéo a été réalisé puis visionné. Ces images montraient alors les femmes effectuant leurs tâches quotidiennes dans les champs. Lors de ce feedback, ces derniers ont été rejetés du point de vue de leur adéquation pour les femmes, puisque considérés comme des activités inappropriées pour elles, qui sont «faites pour les activités du foyer et pour d'autres, plus délicates » (D’Ávila Neto 2002). L'essai provocateur de Donna Haraway, Savoirs situés, regorge de réflexions importantes, aidant à comprendre la question :

«(...) Des savoirs situés demandent que l'objet de connaissance soit vu comme un acteur et un agent, pas comme un simple écran ou un terrain ou une ressource (Harraway 2007 : 130) (...) La description du monde « réel » ne dépend plus alors d'une logique de la " découverte ", mais d' une relation sociale forte de « conversation » (...) Les codes du monde ne restent plus immobiles à attendre qu' on les déchiffre. » (Haraway 2007 : 131)

« Ma manœuvre simple (...) n'est pas évidemment pas neuve dans la philosophie occidentale, mais elle prend un tranchant féministe particulier quand elle est liée à la question de la science dans le féminisme et aux questions connexes du genre comme différence située de l'incorporation femelle. Les écoféministes ont peut-être été celles qui ont le plus travaillé à produire une version du monde comme sujet actif et non comme simple ressource cartographiée et confisquée par les projets bourgeois, marxistes ou mâles. » (Haraway 2007 : 131)

Il est intéressant d'accompagner la pensée féministe de Donna Haraway, car elle se révèle très proche de la pensée de la « colonialité », telle que définie par les auteurs latinoaméricains qui traitent de ce sujet.

\section{Traduire les invisibilités sociales : les femmes et la nature en chair et en os}

Arturo Escobar, pour parler des difficultés vécues par les projets visant de nouvelles voies de la connaissance, tels que celui entrepris par un groupe de penseurs latino-américains, à propos de la modernité et de la colonialité, mentionne l'absence de trois thèmes : (1) la question entre les sexes, (2) la question de la nature et de l'environnement, (3) la question de la nouvelle alternative économique imaginaire. Pour Escobar, ajouter ces dimensions « serait comme ajouter la chair et le sang », c'est-à-dire de la matérialité à ces discussions, qui passent par des questions de race et de genre, au-delà des économies alternatives, basées sur les politiques de lieu. Dans le même sens de matérialisation de questions, jusqu'ici traitées conceptuellement ou d'une manière éloignée de la pratique, Rose Braidotti parle, en matérialisant également le féminin, des « femmes de chair et d'os » (Braidotti 2000).

Voilà ce qui constitue peut-être notre désir (paradoxal ?) face à la nature. La figure de " Grande Mère », pour symboliser la " grande » Nature, apparait comme une appartenance filiale, une recherche dans la « chair et le sang » de la nature de ce qui nous relie 
au cosmos. Moscovici, dans ses réflexions sur l'écologie politique, n’oublie pas cet aspect : «L'éternel retour à la nature, vu sous l'aspect du désir, signifie le retour de tous les corps dans le corps des corps, la terre où chacun se sent chez soi, notre oikos depuis les origines.» (Moscovici 2002 : 181)

Expliciter la visibilité sociale implique d'examiner des relations de pouvoir complexes. Nous pouvons les observer dans la formation du Brésil colonial et de ses mécanismes d'identité culturelle. Le Brésil colonial est un ensemble complexe de relations fondées sur l'autorité et la domination autour d'un « monde seigneurial ». La tradition sociologique brésilienne parle de " pouvoir domestique », de " pouvoir seigneurial » et de « pouvoir aristocratique» (Fernandes 1976). Nous constatons que dans ce monde colonial, aux « sources de notre système » patriarcal, l'autorité du patriarche était incontestable dans la domination de la famille, des femmes, des enfants et des esclaves. C'est dans ce même monde d'autorité patriarcale qu'est engendrée l'exaltation de la Vierge Marie. Associée aux « vertus naturelles » des femmes - obéissantes et soumises - elles devaient demeurer vierges jusqu'au mariage avec un homme, ou jusqu'au mariage mystique avec le Christ, destin de celles qui ne se mariaient pas ${ }^{3}$. Au contraire, il existait une mythification de la sexualité des femmes afro-descendantes. Ainsi, nous avons le pouvoir de la vierge, associé à la femme blanche, aux côtés du pouvoir de l'exaltation de la sexualité, quand « le sexe était plus que du sexe », tel que mentionné par les chroniqueurs de l'époque, plus particulièrement à propos des afro-descendantes, mulâtresses (D`Ávila Neto 1994). Bien qu’appelés « pouvoirs », ils ne possédaient pas le poids de la sphère politique, ils appartenaient à la sphère de l'imaginaire social. Il est important d'observer que la virginité ou « le sexe intouché », ou son " plein exercice », possèdent des résonances au niveau de l'image des femmes, idéalisées avec des pouvoirs magiques, que ce soit en tant que sainte béatifiée, ou en tant que sorcière diabolique. (D`Ávila Neto, Pires, 2000; Dorlin, 2006).

Il ne s'agit pas ici de dichotomiser le monde patriarcal et le monde non patriarcal. L'objectif est de parcourir les trajectoires d'identification, qui servent d'appui à la " femme nature ", dans notre monde colonial et comment ce contexte se reflète dans le Brésil contemporain, au niveau des conditions défavorables que nous rencontrons, à l'intersection de la race/sexe/classe. Pour démontrer l'intersection " race/couleur/classe » et le statut socioéconomique combiné avec le sexe, nous analyserons brièvement les signes visibles des inégalités et injustices sociales que révèlent nos statistiques, que ce soit du fait d'une mauvaise répartition ou de par la négation d'une représentativité et d'une reconnaissance culturelle (Fraser 2007).

${ }^{3}$ Indépendamment de la vocation religieuse, les familles envoyaient au couvent celles qui étaient « déshonorées », qui avaient perdu I'honneur, c'est-à-dire, la virginité. 
En 2006, la population brésilienne était composée à 49,7\% de personnes se déclarant blanches et à 49,5\% - correspondant à 93,1 millions de personnes - de noirs et métis 5 . $0,3 \%$ se sont déclarés de couleur ou de race indigène et $0,5 \%$ de couleur jaune. Entre 1995 et 2006, la population noire et afro-descendante est passée de $45 \%$ à 49,5\%. En 2006, alors que $8 \%$ de la population blanche vivait en dessous du seuil de pauvreté, cet indice, parmi la population afro-descendante, était de 18,8\%. Au niveau de la pauvreté absolue, 29,6\% étaient blancs et 69,8 étaient noirs ou métis. Le revenu mensuel moyen du travail principal des hommes blancs, dans l'ensemble du pays, équivalait à R\$ $1164,00^{6}$. Cette valeur, la même année, était 56,3\% supérieure à la même rémunération obtenue par les femmes blanches (R\$ 744,71) ; 98,5\% supérieure à celle gagnée par les hommes noirs \& métis $(\mathrm{R} \$ 586,26)$ et $200 \%$ supérieure par rapport à celle reçue par les femmes noires \& métisses ( $\mathrm{R} \$ 388,18)$, renforçant ainsi l'inégalité classique de genre et de race.

En ce qui concerne l'accès à l'éducation, l'analphabétisme entre les personnes de 15 ans ou plus, parmi les noirs et leurs descendants, en 2006, était proportionnellement supérieur de $12,4 \%$ par rapport à celui des blancs. La période moyenne de scolarisation des femmes blanches est de 6,6 années, contre 4,5 années pour les femmes noires. À l'école primaire - enseignement déclaré obligatoire - le pourcentage, qui est de $95,9 \%$ et $95,7 \%$ pour les hommes et femmes blanches, atteint $94 \%$ pour les hommes noirs et $94,5 \%$ pour les femmes noires et métisses. Dans l'enseignement secondaire, qui suit l'école primaire, la proportion est de $55 \%$ et de $63 \%$ pour les hommes et les femmes blanches et de, respectivement, $32,7 \%$ et $43,4 \%$ pour les hommes et femmes de couleur de peau noire et métisse. En ce qui concerne les données, les femmes de peau " sombre » présentent des indicateurs plus importants que les hommes du même groupe, dans les deux niveaux d'enseignement. Cependant, ces femmes n'atteignent pas des indices plus élevés que celui des hommes blancs. La population économiquement active (PEA) des femmes noires et métisses dispose d'un accès plus difficile à la sécurité sociale, puisque $65 \%$ du groupe n'a pas accès à cette protection fournie par l'État.

L'étude révèle également que le travail domestique est plus occupé par des femmes que par des hommes, avec une incidence plus élevée parmi les noirs et métis. Il s'agit d'une part très importante de la main-d'œuvre féminine, dans une activité peu valorisée, et qui ne dispose pas des mêmes droits sociaux et du travail, comme l'assurance chômage, par exemple. Dans les statistiques de journée de travail des deux sexes, les hommes atteignent des indices supérieurs à ceux des femmes, puisque le travail relatif aux tâches domestiques, telles que prendre soin de la maison et des enfants, n'est pas repris dans les statistiques officielles. Il s'agit d'un « travail invisible », qui n'est pas in-

\footnotetext{
${ }^{4}$ IBGE - Institut Brésilien de Géographie et Statistique (Projet National d'Échantillonnage par Domicile PNAD). Les statistiques intègrent un apport annuel d'inégalités raciales au Brésil, organisé par Paixão et Carvano (2008).

${ }^{5}$ Dans les statistiques » negros » (noirs) et « métis » (personnes s'étant délarées métisses).

${ }^{6}$ Correspondant à 450 euros, approximativement (oct/2012) ; salaire minimum national équivalant à 250 euros.
} 
tégré dans les statistiques de Population économiquement active (PEA) ou dans les indices socioéconomiques. La prestation de services informels est la seule alternative disponible pour les femmes des régions pauvres, qui émigrent vers les régions plus riches du Brésil, à la recherche de meilleures conditions de vie et d'opportunités de travail. Il existe un important contingent de femmes employées en tant que « domestique », exerçant les fonctions de cuisinière, nourrice (pour prendre soin des enfants) et de femme de ménage, c'est-à-dire toutes les sortes de tâches domestiques quotidiennes, et qui travaillent en général pour les classes plus privilégiées. Le cycle de « travail invisible » est répété car, malgré une faible rémunération, il se déroule fréquemment sans statistiques, puisque l’informalité y est presqu'une règle. Le travail de ces femmes demeure, dans ce cas-ci, invisible, de par le contexte de l'économie. La croissance du travail rémunéré des femmes - en tant qu'une des conséquences de la mondialisation - est produite, dans les faits, par deux groupes de femmes : celles qui travaillent hors de la maison et qui ont besoin d'une " employée domestique », et celles qui restent à la maison pour effectuer les tâches « naturelles » de la femme. Les femmes socio-économiquement plus favorisées ont besoin que le travail domestique soit réalisé par d'autres femmes. Celles-ci, de leur côté, moins favorisées, ont également besoin de disposer de l'appui de leurs proches - frères et sœurs plus âgés, oncles, tantes, grands-parents et voisins, ou même de crèches et de services communautaires précaires - pour prendre soin des enfants, leur permettant ainsi d'exercer leurs fonctions dans les emplois domestiques, ou même, très fréquemment, dans des " emplois formels ", mais avec de bas salaires, comme dans les usines, industries, commerces, magasins, entreprises, hôpitaux, écoles et même, parfois, dans les services publics.

Le projet « modernité-colonialité », tel qu'il fût traité par Arturo Escobar, inclut une nouvelle perspective à propos des subalternes, en particulier par rapport à la manière dont ils ont été représentés, comme s'ils constituaient une catégorie unique homogène. L'auteur attire l'attention sur la nécessité de reconnaissance de la multiplicité de ces groupes. Cela signifie reconnaître que les subalternes doivent affronter des problèmes très différents, par exemple, dans le cas des femmes : la violence, les droits de reproduction, la sexualité et l'identité, souvent associés à la nature et aux notions essentialistes. À ce sujet, la conception de genre est transversale, c'est-à-dire que la femme ne constitue pas une catégorie unique ; elle recouvre des différences générationnelles, ethniques, de capital culturel et socioéconomiques, entre autres. Cette constatation des différences est cruciale, pour empêcher qu'une fausse vision globale masque les contradictions en jeu dans les relations de force et de discrimination ( $D^{\prime}$ Ávila Neto 2004). Les discriminations raciales et sexuelles sont des facteurs importants dans les analyses des conditions de pauvreté et d'inégalités sociales, en Amérique latine.

«Les féministes latino-américaines ont souligné le fait que les femmes sont aussi l'Autre de la modernité. Au-delà des discussions sur la division du travail et de l'épistémologie, ce fait a eu des conséquences visibles pour l'analyse des processus clés de la colonisation, y compris la construction de la nation, de la race et des formations eurocentriques patriarcales. » (Escobar $2003: 75$ ) 
L'épistémologie féministe, en fonction d'une position de revendications politiques, à propos des inégalités des sexes, mène à des études des idéologies et des codes culturels, qui renforcent les conditions de subordination et celles des espaces de représentation des femmes. La théorie féministe permet l'accès à un univers qui a été réduit au silence, ou même effacé des archives historiques ; le féminisme peut réexaminer les moyens de connaître le passé, pour la reconstruction d'une nouvelle base de connaissance. Ceci signifie qu'une critique féministe postcoloniale devrait prendre en considération les jeux de pouvoir entre hommes et femmes de la société patriarcale brésilienne, ce qui comprend la domination et l'exploration du corps - territoire - féminin, associé au corps - territoire - nature. « Il existe des différences dans la manière dont les groupes subalternes sont objets de pouvoir, ou sujet « d'agentivité7 »(Escobar 2003: 72). C'est, quelque part, une manière d'encourager les études qui se proposent de montrer de nouvelles perspectives de la femme, sans être prisonniers de la logique de contre-argumentation, puisque, dans la perspective postcoloniale, cette question est relationnelle. En établissant une corrélation entre les études de la femme et l'écologie, Escobar cherche à démontrer que ces questions sont associées au sein du projet de domination de la nature et des femmes:

« II est difficile pour ceux qui n'ont pas l'habitude de penser en termes d'écologie, de comprendre que la crise environnementale actuelle n'est pas seulement une crise généralisée, mais peut-être la crise centrale et la limite pour le capital aujourd' hui. II est plus facile d'accepter l'idée que la modernité est structurée sur la séparation entre nature et culture (...). »(Escobar 2003 : 77)

La discussion en elle-même, au sujet des politiques macro-économiques et sociales, également toujours maintenues séparées les unes des autres, se présente comme un obstacle à la véritable solution pour une réelle transformation. Il manque à ces politiques, non seulement une justice de genre et de race, mais aussi une grammaire morale, qui assurerait une justice sociale et une reconnaissance (Fraser 2007; Durano et al. 2009). De sévères critiques sont émises à propos de cette lacune par le mouvement « Alternatives de développement pour les femmes dans une nouvelle ère » (DAWN, sigle en anglais), argumentant que les politiques sociales sont élaborées pour traiter les chocs/crises comme externes par rapport au système de production et non pas comme si elles étaient générées par le système lui-même. Cette situation s'aggrave, en ce qui concerne les femmes, à partir du moment où, dans une société capitaliste de travail organisé, la contribution de travail reproductif et productif des femmes n'est pas reconnue comme un générateur de richesse, ou même, en tant que travail (Durano et al. 2009).

Au Brésil, par exemple, l'accès au système public de santé et aux soins est, pour un nombre considérable de personnes alitées, plus pauvres ou appauvries, faible et insuffisant en regard de leurs nécessités. De ce fait, le soin vis-à-vis des personnes âgées ou

\footnotetext{
${ }^{7}$ Néologisme venant de l'anglais agency pour indiquer la capacité d'autonomie des sujets.
} 
des malades en incapacité de travail, devient presque toujours une tâche familiale destinée aux femmes, qui sont, par " nature », les personnes prenant soin, reproduisant une forme de continuité de la maternité et du soin des enfants. Même en considérant que cela puisse constituer un choix de la part de quelques femmes, ou un « trait culturel » désirable, accepté par les autres, il s'agirait toujours d'un poids, de par l'augmentation de la charge de travail de ces femmes. Cette situation est d'autant plus discutable, en fonction d'une politique néolibérale de l'État, à partir du moment où celui-ci prétend se libérer du fait de fournir des équipements nécessaires aux soins et au bien-être. Ce scénario est un défi et est marqué par les hiérarchies de genre, de race, de classe sociale et de situation géographique, entre autres. Il constitue une immense mosaïque d'inégalités sociales au Brésil, et peut être également observé dans divers pays d’Amérique latine.

\section{L'autre de la nature : les femmes et les autres sans-âme}

Dans la Conquête de l'Amérique, Tzvetan Todorov, en faisant référence à la colonisation des Aztèques du Mexique, indique que le « Nouveau Monde » a été proposé en tant que lieu de l'altérité. Les indigènes, considérés comme inférieurs, sont assimilés, en même temps, aux femmes et aux animaux. Les peuples autochtones sont considérés comme primitifs, par rapport aux Espagnols, qui leur sont supérieurs, puisqu'ils possèdent une âme (en plus du corps).

« Il est bien entendu révélateur de trouver les Indiens assimilés aux femmes, ce qui prouve le passage facile de l'autre intérieur à l'autre extérieur (puisque $c^{\prime}$ est toujours un homme espagnol qui parle) ; (...) les Indiens faisaient une distribution symétrique et inverse : les Espagnols étaient toujours assimilés aux femmes, par le biais de la parole (...). » (Todorov 1982 : 159)

Pour l'auteur, le significatif est la solidarité des projections et non la priorité de l'une par rapport à l'autre, c'est-à-dire qu'il n'importe pas que nous projetions l'image des étrangers sur les femmes, ou vice-versa. Pour le conquistador, c'est l'existence, ou non, d'une âme qui est l'aspect révélateur. "La mise en équivalence de ces oppositions avec le groupe relatif au corps et à l'âme est également révélatrice : avant tout, l'autre est notre corps même ; d'où aussi l'assimilation des Indiens comme des femmes aux bêtes, à ceux qui, bien qu'animés, sont sans âme » (ibid.159-160). Boaventura Santos, penseur postcolonial, quant à lui, explique que deux processus différents peuvent se produire dans le cadre de la reproduction des identités dominantes : le premier, au travers de la négation totale de l'autre, et le second, de par le conflit vis-à-vis de l'identité subalterne de l'autre. « (...) Par exemple, l'identité dominante et même matricielle de la modernité occidentale - Prosper/Caliban, civilisé/sauvage, noir/blanc, homme/femme - s'est reproduite par le premier processus et ensuite dans l'autre »(Santos 2006: 250). On peut ainsi mieux comprendre les questions ayant trait à la place de la femme et de la nature dans l'imaginaire du patriarcalisme colonial : tant la femme que la nature étaient des 
propriétés, qui devaient être soumises au juge seigneurial. Les thématiques de l'écoféminisme et de l'écologie politique féminine sont replètes d'exemples et de théories, à propos du même aspect.

Il est nécessaire de rappeler que les Indiens n'avaient pas d'âme, parce qu'ils n'étaient pas chrétiens, et seule la conversion religieuse pouvait leur donner une condition moins bestiale, aux yeux des conquistadors. Dans l'imaginaire multiforme, les femmes sont considérées comme des biens (dans le sens de propriété) et leurs prédicats et vertus, ce qui est « inné » ou « naturel » chez les femmes, vont renforcer une fois de plus l'essentialisme. Dans la société patriarcale brésilienne, il existe également une mise en emphase des vertus et prédicats " corporels et spirituels », distinguant blanches et métisses, donnant un caractère racial à l'essentialisme. D'un autre côté, les Indiens demeurent liés à la nature et à l'état brut, dans une forêt emplie d'animaux dangereux, alors que l'imaginaire oscille entre l'utopie du «bon sauvage » idyllique et l'image du cannibalisme effrayant. Sans aucun doute, dans la façon de comprendre la nature, il existe une profonde ligne de démarcation entre l'imaginaire de l'indigène et celui de l'homme blanc, ou « conquistador », en mission d'évangélisation:

« La nature apparait être l'autre côté de la différence coloniale, avec certaines natures (coloniales, du tiers-monde, des corps des femmes) (...), situées dans l'extériorité du monde eurocentriste masculin. La crise environnementale montre les limites de la rationalité instrumentale moderne. Elle reflète l'échec du régime moderne, au travers de l'articulation de la biologie et de l'histoire, maintenue par la capitalisation de la nature et du travail. » (Escobar, 2003: 78)

Donna Haraway attire l'attention sur ce même point : « Il a semblé pratiquement impossible d'éviter le piège d'une logique de l'appropriation et de la domination incluse dans le binarisme nature/culture (...) dont il fait partie la distinction sexe/genre » (Haraway 2007:130). Ce piège est engendered ${ }^{8}$, de telle façon que l'on peut observer à l'académie des recherches qui prétendent prouver l'égalité des sexes, en travaillant, par exemple, sur la comparaison des aptitudes de jeunes filles et de jeunes garçons pour les mathématiques ou les « sciences dures ». Il ne s'agit pas d'éliminer ces recherches ni d'autres, tributaires de concepts aristotéliciens. L'important est d'insister sur la partialité de la science/connaissance et de rejeter l'essentialisme. Il est nécessaire de faire plus de progrès à propos de la «partialité » de l'objectivité:

" Nous avons besoin du pouvoir des théories critiques modernes sur la façon dont les significations et les corps sont fabriqués, non pas pour dénier significations et corps, mais pour vivre dans les significations et des corps pour qu'ils aient une chance dans l' avenir. » (Haraway 2007 : 113)

\footnotetext{
${ }^{8}$ Fait référence à gender ou genre.
} 


\section{Pour décoloniser le corps (des femmes) et de la nature}

Le rôle des chercheurs pourrait être celui de la tradition culturelle, par-dessus tout, transculturelle, en supposant l'impossibilité de l'universalisme, et dans le sens de recréer des frontières. La traduction de la culture des subalternes a été l'objet d'innombrables discussions, dans la littérature postcoloniale et dans une grande partie de la critique féministe contemporaine.

Le texte de Spivak, l'Indienne enracinée aux États-Unis, "Can the subaltern speak?" " est devenu un classique, en tentant de proposer l'impossibilité de l'expression des subalternes, nécessaire pour sa légitimation (Fanon 1972). La question est d'ailleurs traitée sous différents angles, tels que celui présenté par Stuart Hall, et qu'il appelle «nouvelles ethnicités » (1996 [1989]). Ces groupes, issus de la diaspora, remettent en question les représentations figées qui leur sont imposées, au nom de leurs diverses expériences sociales et subjectives, en recherchant des moyens d'organisations alternatives des communautés ethniques (Santos $2006: 15$ ).

Il n'est pas possible d'aborder cette question sans tenir compte de l'intersection du sexe, de la race et de la classe, afin de mettre en évidence "l'épistémologie de la domination », comme le proposent les féministes Elza Dorlin, en France et Jurema Werneck, au Brésil, lorsqu'elles discutent à propos du féminisme du sud, ou du black feminism.

« Nous devons expliciter l'impossibilité pratique de dissocier le patriarcalisme du racisme, du colonialisme et du capitalisme - tout ceci fait partie d'un même " sac », celui de la domination occidentale sur toutes les autres régions du monde. Ces systèmes de domination ne sont pas structurés comme des chapitres ou des hiérarchies, au contraire, ils fonctionnent simultanément sur les femmes et, parfois, fort douloureusement. » (Werneck 2007 : 100)

L'énoncé centré sur la manière occidentale a défini et définit encore « les femmes du sud ». Cette opposition au féminisme blanc occidental, constitue le motif des plaintes de la part du féminisme de vision non eurocentriste, tel que le black feminism, le féminisme des chicanas « new mestizas », borderlands, etc. Il existe une convergence entre divers auteurs, qui soulignent la nécessité que soit traduite au travers du corps et des expériences, l'énonciation de groupes minoritaires ou subalternes. Ce n'est pas une question de substitution du langage verbal par le langage corporel/gestuel. Il s'agit de nouveaux modes d'énonciations prospectives, plus fluides, plus hybrides, plus proches des utopies émancipatoires, comme le préconise Boaventura Santos, et qui pourraient être contenus dans la métaphore du concept de frontière :

«J'ai utilisé la notion de frontière, plus dans le sens d'extrémité (frontière), que dans le sens de zone de contact (borderlands), mais, en tout cas, je cherche, par le biais de la notion de frontière à signifier le déplacement du mot et des pratiques du centre vers la périphérie. Je propose une phénoménologie de la marginalité basée sur une utilisation sélective et instrumentale des traditions, dans l'invention de nouvelles formes de sociabilité (...). » (Santos 2006 : 241)

${ }^{9}$ Publié en France sous le titre « Les subalternes peuvent-elles parler?». 
Quelles sont les nouvelles formes ? Comment les (ré)inventer ? Comment les traduire ? Rose Braidotti (2000) indique que le point de départ du projet de la différence sociale demeure, par-dessus tout, sa volonté politique d'affirmer la spécificité de l'expérience corporellement féminine, en rejetant la différence sexuelle au niveau du sujet désincarné, c'est-à-dire, post-moderne et anti-essentialiste. Francis souligne que ces «stratégies identitaires (...) innervent le récit postmoderne occupé par le démantèlement du sujet positiviste, phallocentrique » (Cecília Francis, 2002 : 133). Braidotti (2000) commente que, selon Teresa de Lauretis, nous devons reconnaître qu'il existe une différence entre la représentation des femmes (les femmes en tant qu'imago culturel) et l'expérience en tant que femme (femmes en tant qu'agents du changement). Dans sa proposition, qu'elle appelle la position féminine nomade, elle reconnait les différentes manières de représenter la femme, faisant valoir que cela est nécessaire pour éviter des divisions au sein de la pratique féministe. Ainsi, l'identité femme ne doit pas être définie comme le non humain, ou comme une essence. La nouvelle figuration du sujet féminin, soutenue par Braidotti, est un projet transdisciplinaire, proposant « un échange plus efficace entre les artistes et les esprits théoriques, académiques et créatifs.» (Braidotti, 2000).

« La vision que je propose (...) implique que le corps ne puisse se capter ou se représenter pleinement : il excède la représentation. (...) Pour moi, l'identité est un jeu aux multiples aspects, fracturés entre eux-mêmes ; il est « relationnel », car il existe une nécessité d'un lien avec l'» autre », il est rétrospectif, car il se fixe en vertu de la mémoire et des souvenirs (...). » (Ibid. : 195)

Le corps, au même titre que la langue, est aussi une expression d'un lieu de pouvoir. Différents auteurs suggèrent la nécessité d'introduire un registre visuel, pour capter le langage corporel en tant que « discours » des femmes, particulièrement ceux qui concernent les groupes culturellement défavorisés ou minoritaires. L'idée centrale est que le corps des femmes, dans une société patriarcale telle que la nôtre, constitue un point de convergence des stratégies de pouvoir. Dans les documents sociohistoriques de la culture brésilienne, nous percevons que les femmes du Brésil colonial ont appris à se taire, face aux plus âgés, tels que leur père, leur mari ou même leur patron, l'homme étant le seigneur absolu de son domaine, qui inclut propriétés, esclaves et famille. Les femmes silencieuses devant l'autorité du seigneur parlent au travers de leurs corps, en baissant la tête. Cecília Francis (2002) explique que, pour capturer le langage postcolonial, le personnage du roman de l'écrivaine algérienne Leila Sebbar-Sherazade utilise la fonction visuelle, en montrant comment va se configurer son « agentivité » par rapport à sa propre identité narrative :

«Ce programme d'apprentissage aléatoire sous-tend chez elle l'émergence des compétences scriptrices, ce qui présuppose une transformation du sujet, surtout en ce qui concerne ses modalités de voir et de comprendre. On assiste, en effet, à l'affranchissement graduel - sexuel et culturel - du sujet féminin métissé au moyen d'un parcours visuel impliquant le renversement dans l'ordre des hiérarchies du sujet regardé/sujet regardant. » (Ibid. : 145) 


\section{Conclusion}

Dans un travail de sensibilisation de femmes, appartenant à des groupes défavorisés, sur le plan du développement culturel, en suscitant un retour par le biais des images vidéo (videofeedback) des jeux dramatiques inspirés par le théâtre de l'opprimé (Augusto Boal, 1998), nous avons observé d'intéressantes performances au niveau du discours corporel. Nous donnerons l'exemple d'une session de groupe, qui fût réalisée en utilisant comme thème les relations entre patrons et employés domestiques. Dans un des jeux, les femmes de ménage elles-mêmes représentaient un patron avec sa famille, face à une femme de ménage, qui était réprimandée. Lors de la session de videofeedback de cet exercice, nous avons demandé une appréciation des scènes qui avaient été interprétées. Dans un premier temps, se limitant à l'expression langagière, les chercheurs n'avaient pas compris la scénographie du corps. Ils ont ainsi supposé que le dialogue représenté (au sens de la représentation scénique), entre le patron et l'employée, empli de préjugés et de discrimination, devait fonctionner comme une provocation pour exprimer les relations de domination/subalternité. Cette hypothèse ne s'est pas vérifiée. Durant le visionnage vidéo, ce ne sont pas les dialogues qui ont le plus attiré l'attention du groupe, mais bien la position de la tête de la femme de ménage maintenue baissée, qui se révéla être le facteur le plus significatif et le plus parlant. Les échanges ont été pratiquement abandonnés durant la session de videofeedback. Il aurait pu être une sorte d'imitation du quotidien, sans importance. C'est le corps courbé, la tête basse, dénonçant une forme corporelle de domination et d'oppression, qui suscita les commentaires des femmes présentes. Pour les chercheurs, la scénographie du corps ne fut pas perçue ou vue, dans un premier temps, car ils étaient limités par l'expression langagière, par ce qui est fréquemment collecté dans les enquêtes de terrain (D’Avila Neto, Baptista 2007).

Peut-être pourrions-nous, dans nos recherches, apprendre à considérer la vue en tant que savoir toujours situé. Appendre (ou réapprendre) à voir et à répondre à certaines questions de Haraway : «Comment voir ? D'où voir ? Pourquoi voir ? Avec qui voir ? (...) Qui arrive à soutenir plus d'un point de vue ? Qui est borné ?» (2007 : 123). Peut-être pourrions-nous et devrions-nous travailler avec l'objectif d'ouvrir de nouvelles frontières : les frontières peuvent exclure, subjuguer et définir l'autre comme différent, étrange, dangereux, mais les frontières peuvent également recréer, subvertir, traduire de nouvelles subjectivités. Nous pouvons supposer que la logique de l'oppresseur/colonisateur s'intègre avec l'identité de l'opprimé/colonisé et vice-versa et, d'une manière ou d'une autre, les récits de ces femmes possèdent de multiples facettes, qui sont contées par les voix de leurs corps et de leurs mémoires colonisées (D`Ávila Neto, Batista 2007). 


\section{Bibliographie}

Anzaldúa G. (1987), Borderlands/La Frontera: the new mestiza. San Francisco : Spinsters/Aunt Lute

Bahri D. (2006), « Le Féminisme dans/et le Post-Colonialisme », In: Lazarus,N. (Org.) Penser le Post-Colonial: une Introduction Critique. Paris : Amsterdam.

Beck U. (2001), La Société du Risque-Sur la voie d'une autre modernité, Paris : Aubier.

Boal A. (1988), Jogos para atores e não-atores, Rio de Janeiro : Civilização Brasileira.

Braidotti R. (2000), Sujetos Nomades, Buenos Aires : Paidós.

Butler J. et Spivak G.C. (2007), L’Etat Global, Paris: Payot.

Canclini N. G. (2008), Culturas Híbridas: estratégias para entrar e sair da modernidade, São Paulo : Ed.USP.

D`Ávila Neto M. I., Durand Delvigne A. et Nazareth J. (2012), « Immigrant Women: Feminism, Recognition and Social Justice in France and Brazil », In : Bonifacio G. (Org.), Feminism and Migration: Cross-cultural Engagements, London, New York: Springer.

D’avila Neto M. I., Baptista C. (2007), « Páthos e o sujeito feminino: considerações sobre o processo de construção narrativa identitária de mulheres de grupos culturalmente minoritários », Revista Pesquisas e Práticas Psicossociais, vol. 2, pp. 2 10.

D’avila Neto M. I. et Nazareth J. (2005), «Globalization and Women's Employment », Peace Review - A journal of social justice, vol.17, n²/3, pp. 215-220.

D’Ávila Neto M. I. et Pires C. (2000), « Empowerment or powersharing? Considerations based on gender equity research in Brazil », In : M. L. Kearney (org.), Women, Power and the Academy: from rhetoric to reality, Nova York: Berghahn Books.

D`Avila Neto M. I. et Vasconcelos N. (1993), « Ecologia, Feminismo e Desenvolvimento », Série Documenta EICOS/UFRJ, n¹, (épuisé).

D`Avila Neto M. I. (1993), Participation Ambiguë, n.spécial Sciences sociales et développement -Le savoir et le politique Courrier de la Planète, $\mathrm{n}^{\circ} 74$.

D’Avila Neto M. I. (2002), As Bordadeiras /Les Brodeuses , vidéo 39 minutes, Copyright UNESCO, pour le projet Participation et Développement Durable Nouvelles Stratégies, Vieux Défis. Paris : UNESCO (extraits disponibles en ligne www.eicos.psycho.ufrj.br).

D`Avila Neto M. I. (1999), « Ecofeminismo : Horizontes Contemporâneos », Documenta EICOS, $\mathrm{n}^{\circ} 8$, Rio de Janeiro : UFRJ. 
D`Avila Neto M. I (1995), « Mulheres, Cultura e Desenvolvimento », In: D`Ávila Neto M.

I. (Org.), Desenvolvimento Social: desafios e estratégias, Rio de Janeiro : UNESCO Chair/UFRJ/FINEP, vol. 2, pp. 203-226.

D’Avila Neto M.I (1994), O autoritarismo e a mulher : O jogo da dominação macho-fêmea no Brasil [1980], Rio de Janeiro : Artes \& Contos.

Dorlin E. (2006), La Matrice De La Race - généalogie sexuelle et coloniale de la nation française, Paris : La Découverte.

Durano M., Francisco G. et Sen G. (2009), « Reclaiming Institutional and Policy Space Amidst Crisis Development », Society for International Development Development Alternatives with Women for a New Era, 52(3), pp. 334-337.

Escobar A. (2003), « Mundos Y Conocimientos De Outro Modo-El programa de Investigacion de Modernidad/colonialidade Latino Americano », Tabula Rasa, n001, pp. 51-86.

Escobar A. (2005), Más allá del tercer mundo: globalización y diferencia, Bogotá : ICANH Universidad de Calca.

Fanon F. (1971), Peau noire, masques blancs, Paris : Seuil.

Fernandes F. (1976), A Sociedade Escravista do Brasil (The slavish society of Brazil), paper presented in the Academy of Sciences. New York.

Ferry L. (1992), Le nouvel ordre écologique, Paris : Grasset.

Francis C. W. (2002), "Énonciation, discours et stratégies identitaires. Une phénoménologie de l'altérité dans l’œuvre de Leila Sebbar », In : Ouellet P. et al. Identités narratives, mémoire et perception, Québec : Presses universitaires de Laval.

Fraser N. (2007), " Mapeando a imaginação feminista: da redistribuição ao reconhecimento e à representação ", Revista De Estudos Feministas. vol. 15, n²2, maio/agosto.

Grosfoguel R. et Mignolo W. (2008), « Intervenciones Descoloniales : una breve introdución », Tabula Rasa, n9, pp. 29-37.

Grosfoguel R. (2008), « Para descolonizar os estudos de economia política e os estudos pós-coloniais: Transmodernidade, pensamento de fronteira e colonialidade global », Revista Crítica De Ciencias Sociais, n80: pp. 115-147.

Gruzinski S. (2006), A guerra das Imagens, Rio de Janeiro : Companhia das Letras.

Hall S. (2006), « Pensando a Diáspora: Reflexões Sobre a Terra no Exterior », In : Sovik, L. (Org), Da diáspora - identidades e mediações culturais, Belo Horizonte : Ed.UFMG. 
Hall S. (1996), « New Ethnicities [1989] » In Morley D. et Kuan-Hsing C. (org), Critical Dialogues in Cultural Studies, London and New York: Routledge, pp. 441-449.

Haraway D. (2007), Manifeste cyborg et autres essais - Sciences-Fictions-Féminismes, Paris : Exils.

Haraway D. (1988), « Situated Knowledges : the Science Question in Feminism and the Privilege of Partial Perspectives », Feminist Studies, vol. 14 n³, pp. 575-599.

Lugones M. (2008), « Colonialidad y Género », Tabula Rasa. Bogotá - Colombia, n.9, pp. 73-101.

Merchant C. (1980), The Death of Nature, New York : Harper and Row.

Mies M.; Shiva V. (1999), Ecofeminismo, Lisboa : Piaget.

Mignolo W. (2007), La idea de America Latina-La herida colonial y opcion decolonial, Barcelona : Gedisa.

Miñoso Y.E. (2009) , « Etnocentrismo Y Colonialidad En Los Feminismos Latinoamericanos: Complicidades Y Consolidación De Las Hegemonías Feministas En El Espacio Transnacional », Revista Venezolana De Estudios De La Mujer - Julio/Diciembre 2009, vol. 14, n³3, pp. 37-54.

Moscovici S. (2002), De la Nature pour penser l'écologie, Paris : Metaillié.

Paixão M. et Carvano L. (2008), Relatório das Desigualdades Raciais no Brasil, Rio de Janeiro : Garamond.

Ribeiro D. (1995), O povo brasileiro, Rio de Janeiro : Companhia das Letras.

Santos B.S. (2006), A Gramática do Tempo para uma nova cultura política, São Paulo : Cortez.

Shiva V. (2010), Las guerras del agua: privatización, contaminación y lucro, New York : South End Press.

Spivak G. C. (2010), Pode o subalterno falar ?, Belo Horizonte : Editora UFMG.

Todorov T. (1982), La Conquête de l’Amérique, Paris : Seuil.

Warren K. (1993), « Taking empirical data seriously: an ecofeminist philosophical perspective ", Conference on Human values and Environment, University of Wisconsin, In D’Avila M. I. et Vasconcelos N., " Ecologia, Feminismo e Desenvolvimento », Série Documenta EICOS/UFRJ, n¹, pp. 190-211.

Warren K. et College M. (1991), Hypatia: a journal of feminist philosophy, Special Issue (Ecological Feminism). Spring, vol. 6, nº1. 
Warren K. (1987), « Feminism and ecology: making connections », Environmental Ethics, 9, pp. 17-18.

Werneck J. (2007), « Of Ialodês and Feminists: Reflections on Black Women's Political Action », In : Latin America and the Caribbean Cultural Dynamics, London : SAGE, pp. 99-113. 